\title{
Market Power, Survival and Accuracy of Predictions in Financial Markets*
}

\author{
Patrick Leoni \\ National University of Ireland at Maynooth \\ Department of Economics \\ Maynooth, Co. Kildare, Ireland \\ E-mail: patrick.leoni@nuim.ie
}

Keywords: Market selection hypothesis, Market power, Survival, Asset pricing.

JEL codes: G11, G14.

*Thanks to be added later. 


\begin{abstract}
In a standard General Equilibrium framework, we consider an agent strategically using her large volume of trade to influence asset prices to increase her consumption. We show that, as in Sandroni (2000) for the competitive case, if markets are dynamically complete and some general conditions on market preferences are met then this agent' long-run consumption will vanish if she makes less accurate predictions than the market, and will maintain her market power otherwise. We thus argue that the Market Selection Hypothesis extends to this situation of market power, in contrast to Alchian (1950) and Friedman (1953) who claimed that this selection was solely driven by the competitiveness of markets.
\end{abstract}




\section{Introduction}

Consider economic agents who trade financial assets under uncertainty. The long-standing Market Selection Hypothesis, developed by Alchian (1950) and Friedman (1953), states that agents who do not make accurate predictions about the economic uncertainty are eventually driven out of the market. Part of their explanation for this phenomena stems from the competitiveness of financial markets; i.e., fierce competition among agents leads to the "natural selection" of those having the best forecasts.

Even though recent papers have cast a doubt on this approach (see Blume and Easley (1992) for instance), Sandroni (2000) gives a theoretical framework where this evolutionary view holds. Sandroni describes a model with dynamically complete markets, where agents trade securities competitively and where investment-consumption decisions are endogenous. Sandroni then shows that the agents making the most accurate predictions will eventually dominate the market, and that the agents who do not make accurate predictions are necessarily driven out in the long-run.

A natural question that arises from Sandroni's work is to understand to which extent his results rely on the competitive nature of the market. In other words, we address the following question: will an agent with market power always survive in a financial market, even if she makes less accurate predictions than other agents in the market? 
We answer this question by showing that Sandroni's results still hold when one agent has enough influence on financial markets to strategically affect prices of traded assets. Typical examples of such agents are hedge funds ${ }^{1}$ or central banks influencing interest rates through open market operations. We develop a general equilibrium model, close to Sandroni's, where a strategic agent trades with an arbitrary number of competitive agents aggregated into a representative agent (the market) forming expectations about economic uncertainty. The strategic agent uses her large volume of trade to influence market prices to increase her consumption. As standard in general equilibrium, the strategic use of a large volume of trade allows to indirectly control the price of every traded asset. We then show that, if markets are dynamically complete and some general conditions on market preferences are met, then the agent with market power will vanish in the long-run if she makes less accurate predictions than the market, and will survive otherwise. ${ }^{2}$

The intuition of our result is contained in the following example. Consider an infinite-horizon economy with complete markets, where nature draws in every period the states $\{h, l\}$ in an i.i.d. manner. The probability that nature draws the state $h$ converges to 1 a.s. Consider also two traders, Trader 1 is a price-taker with correct beliefs and Trader 2 is the manipulator believing that the probability that nature draws state $l$ converges to 1 a.s. A natural manipulation for Trader

\footnotetext{
${ }^{1}$ See for instance Hens et al. (2004) for an analysis of this issue and related literature.

${ }^{2}$ In a sense defined later. The same concepts of accuracy of beliefs and survival as in Sandroni (2000) are used here.
} 
2 is to allocate part of her current wealth to increase, in some future history, her demand for consumption in state $l$ and to decrease by the same amount her demand for consumption in state $h$ in this same history. Since markets are complete, Trader 2 can carry out such transactions and she knows that, in this history, the ratio of relative price of consumption in $h$ divided by that in $l$ will increase. This manipulation matches Trader 1' objectives, since this new system of prices allows her, in this history, to increase further her desired consumption in state $h$ and to forego even further undesired contingent consumption in $l$. It is easy to see that the equilibrium strategy to Trader 2 must be such that there is no more profitable manipulation as above in any history, since such manipulations allow the manipulator to increase her contingent consumption in her preferred state while trading away undesired consumption in the other state in the most (subjectively) advantageous manner. However, at the resulting equilibrium prices the manipulator has traded away her consumption in the state that will eventually occur almost surely, and she will thus eventually disappear.

The insight from the above example is central to our result, even if our analysis deals with arbitrary state processes and beliefs. First, the example shows why market power does not change the odds of survival in comparison to the competitive case. Indeed, the manipulator objective is to trade away consumption in state $h$, which she can do at any competitive prices. The manipulator can thus trade away in state $h$ at least as much consumption as she would in the competitive case since she can always enforce such prices, and we already know 
that she will eventually vanish in the latter case. The example also shows why incomplete markets and their potential inability to carry out the above transactions may prevent Trader 2 from vanishing in the long-run, as pointed out in Blume and Easley (2004) for the competitive case. The example also suggests why more assumptions are needed in our price manipulation setting than in the competitive case: one must rule out the possibility that price manipulations generate an infinite wealth to the strategic agent (Assumption 1 presented later), also one must keep the ratio of relative prices bounded (Assumption 2). Those assumptions are on market reactiveness to price manipulations, and they are satisfied by standard market preferences.

Our work thus shows that perfect competition is not a necessary condition to market selection, whereas it appears as a sufficient condition in the Darwinian approach heuristically developed by Alchian. In the same spirit, we show that Pareto-optimality is not a necessary condition for market selection, in contrast to Blume and Easley (2004) that shows that it is a sufficient condition. Indeed, our equilibrium allocations are not Pareto-optimal in general: Stokey and Lucas (1996) Chapter 15 shows that Pareto-optimal allocations and competitive allocations coincide exactly in complete markets, and our set of strategic equilibria differ from the set of competitive equilibria (this appears clearly from Eq. (16), see also Hens et al. (2004) for a discussion of this issue in a related framework). 
In more details, we consider an environment where time is discrete and continues forever. There is an arbitrary number of agents who live forever, one of them being a strategic agent, while the others are usual price-takers facing standard budget constraints. We assume that the price-takers are aggregated into a single representative agent.

In every period, there is a single consumption good available to the agents. A state of nature is drawn from a finite set according to a given probability distribution, which determines the level of endowments of the consumption good for each agent. We assume that endowments are uniformly bounded away from zero and infinity. Every agent forms subjective beliefs about the true probability of the states of nature, and maximizes the expected discounted sum of one-period utility that she gets from consuming the good.

In every history, new markets for consumption good and standard Arrow securities open. We assume that, for every finite history, there exists one Arrow security with strictly positive payoff in this history. This last assumption implies that markets are complete for every strictly positive assets prices. Our results extend to any other asset structure with this property.

The consumption-investment decisions of the strategic agent are constrained to the fact that 1) her own consumption must be affordable, and 2) that market clearing conditions on every market are endogenized. The second part of the problem faced by the strategic agent captures the market manipulation by anticipating the price formation. 
In order to prove existence, we show that the above framework is equivalent to a new one where all future contingent strategic decisions are made in the first period, in a similar fashion as in Debreu (1959), Chapter 9. We need to restrict the set of one-period utility functions of the market to prove existence. This assumption is meant to prevent the strategic agent from accumulating an infinite wealth through price manipulation, and it is satisfied by standard preferences.

In the above environment, all the results in Sandroni (2000) extend to the strategic agent, provided that some restrictions on market preferences are satisfied (standard preferences satisfy them). Say that an arbitrary agent makes accurate predictions if, given enough data, the difference between the agent's beliefs and the true probabilities of the states of nature becomes arbitrary small in the long-run. When all the agents have the same intertemporal discount factor, we show that if the strategic agent makes accurate predictions then she will survive on the market; however there are cases where she will not. For instance, we show that if the representative agent makes accurate predictions and the strategic agent does not, then the consumption of the strategic agent will converge to zero almost surely.

The paper is organized as follows: in Section 2 we describe the model and we make explicit an equilibrium concept with strategic trading; Section 3 contains our main result related to the long-run performances of the strategic agent; Section 4 contains some concluding remarks. Finally, Appendix A contains the proof of existence and Appendix B contains the proof of the main result. 


\section{The model}

In this section, a formal description of the model is given. The following notations are introduced in Sandroni (2000). Time is discrete and continues forever. In every period $t \in N_{+}$, a state is drawn by nature from a set $S=\{1, \ldots, L\}$, where $L$ is strictly greater than 1 . Before defining how nature draws the states, we first need to introduce some notations.

Denote by $S^{t}(t \in N \cup\{\infty\})$ the $t$-Cartesian product of $S$. For every history $s_{t} \in S^{t}(t \in N)$, a cylinder with base on $s_{t}$ is defined to be the set $C\left(s_{t}\right)=$ $\left\{s \in S^{\infty} \mid s=\left(s_{t}, \ldots\right)\right\}$ of all infinite histories whose $t$ initial elements coincide with $s_{t}$. Define the set $\Gamma_{t}(t \in N)$ to be the $\sigma$-algebra which consists of all finite unions of cylinders with base on $S^{t}{ }^{3}$ The sequence $\left(\Gamma_{t}\right)_{t \in N}$ generates a filtration, and define $\Gamma$ to be the $\sigma$-algebra generated by $\cup_{t \in N} \Gamma_{t}$. Given an arbitrary probability measure $Q$ on $\left(S^{\infty}, \Gamma\right)$, we define $d Q_{0} \equiv 1$ and $d Q_{t}$ to be the $\Gamma_{t}-$ measurable function defined for every $s_{t} \in S^{t}\left(t \in N_{+}\right)$as

$$
d Q_{t}(s)=Q\left(C\left(s_{t}\right)\right) \text { where } s=\left(s_{t}, \ldots\right)
$$

Given data up to and at period $t-1(t \in N)$, the probability according to $Q$ of a state of nature at period $t$, denoted by $Q_{t}$, is

$$
Q_{t}(s)=\frac{d Q_{t}(s)}{d Q_{t-1}(s)} \text { for every } s \in S^{\infty}
$$

with the convention that if $d Q_{t-1}(s)=0$ then $Q_{t}(s)$ is defined arbitrarily.

\footnotetext{
${ }^{3}$ The set $\Gamma_{0}$ is defined to be the trivial $\sigma$-algebra, and $\Gamma_{-1}=\Gamma_{0}$.
} 
The posterior probability of $Q$ given a finite history $s_{t} \in S^{t}(t \in N)$, denoted by $Q_{s_{t}}$, is

$$
Q_{s_{t}}(A)=\frac{Q\left(A_{s_{t}}\right)}{Q\left(C\left(s_{t}\right)\right)} \text { for every } A \in \Gamma
$$

where $A_{s_{t}}$ is the set of all paths $s \in S^{\infty}$ such that $s=\left(s_{t}, s^{\prime}\right)$ and $s^{\prime} \in A{ }^{4}$

In every period and for every finite history, nature draws a state of nature according to an arbitrary probability distribution $P$ on $\left(S^{\infty}, \Gamma\right)$.

To conclude this section, we define the operators $E^{Q}$ to be the expectation operators associated with $Q$. Finally, we say that a finite history $s_{t+p} \in S^{t+p}$ follows a finite history $s_{t} \in S^{t}(t, p \in N)$, denoted by $s_{t+p} \hookrightarrow s_{t}$, if there exists $s \in S^{p}$ such that $s_{t+p}=\left(s_{t}, s\right)$.

\subsection{The agents}

In this section, economic agents are described. There is an infinitely-lived agent, the strategic agent, and a market composed of infinitely-lived price-takers behaving competitively. The strategic agent is denoted by $M$ for manipulator, and we assume that the price-takers are aggregated into a single representative agent, denoted by $R$ for representative.

There is a single consumption good available in every period $t\left(t \in N_{+}\right)$. Denote by $c_{s_{t}}^{i}$ the consumption of an agent $i(i=M, R)$ in period $t$, for the history $s_{t} \in S^{t}\left(t \in N_{+}\right)$. In every period $t\left(t \in N_{+}\right)$and in every history $s_{t} \in S^{t}$, every agent $i(i=M, R)$ is endowed with $w_{s_{t}}^{i}$ units of consumption

\footnotetext{
${ }^{4}$ If $Q\left(C\left(s_{t}\right)\right)=0$, then $Q_{s_{t}}$ is defined to be an arbitrary probability measure on $\Gamma$.
} 
goods before the state of nature is revealed to her. We assume that there exist two strictly positive constants $A$ and $B$ such that

$$
A<w_{s_{t}}^{i}<B \text { for every } s_{t} \text { and } i
$$

This last assumption ensures that any agent can secure a strictly positive consumption along any path $s \in S^{\infty}$ by simply staying in autarchy. The aggregate endowment $w_{s_{t}}$, in every history $s_{t}\left(s_{t} \in S^{t}\right.$ and $\left.t \in N_{+}\right)$, is given by

$$
w_{s_{t}}=\sum_{i=M, R} w_{s_{t}}^{i}
$$

In every period $t \in N$, and after the realization of the history $s_{t} \in S^{t}$, the agents trade $L$ one-period securities. Every security $d_{s_{t}}^{j}\left(1 \leq j \leq L\right.$ and $\left.s_{t} \in S^{t}\right)$ purchased in history $s_{t}$ payoffs $d_{s_{t}}^{j}(l)$ unit of consumption good in period $t+1$ if state $l$ is drawn, where $d_{s_{t}}^{j}(l)=1$ if $j=l$ and 0 otherwise. ${ }^{5}$ Let $d_{s_{t}}$ denote the vector $\left(d_{s_{t}}^{1}, \ldots, d_{s_{t}}^{L}\right)$ for every $s_{t}$. The supply of each security is assumed to be 0 in every history.

The price in history $s_{t}$ of security $d_{s_{t}}^{j}(1 \leq j \leq L)$ is denoted by $q_{s_{t}}^{j}$, for every $s_{t} \in S^{t}$ and $t \in N_{+}$. Let $q_{s_{t}}$ denote the vector $\left(q_{s_{t}}^{1}, \ldots, q_{s_{t}}^{L}\right)$ for every history $s_{t}$.

A portfolio $\theta^{i}$ for every agent $i$ is a vector $\left(\theta_{s_{t}}\right)_{s_{t} \in S^{t}, t \in N_{+}}$of quantities held of the $J$ securities in every history $s_{t}$, where $\theta_{s_{t}}=\left(\theta_{s_{t}}^{j}\right)_{j}$ and $\theta_{s_{t}}^{j}$ is the holding of security $d_{s_{t}}^{j}$ in history $s_{t}$. Every agent $i$ has no initial portfolio at date 0 .

Every agent $i(i=M, R)$ does not have any information about $P$, the true

\footnotetext{
${ }^{5}$ Such securities are commonly known as Arrow securities. The choice of this asset structure is solely meant to ensure that, for every vector of strictly positive prices, markets are complete.
} 
probability measure from nature draws the states; however agent $i$ has a subjective belief about nature represented by a probability measure $P^{i}$ on $\left(S^{\infty}, \Gamma\right)$.

Every agent derives some utility in any history from consuming the only consumption good present in the economy. We assume that agent $i(i=M, R)$ ranks all the possible future consumption sequences $c=\left(c_{s_{t}}\right)_{s_{t} \in S^{t}, t \in N_{+}}$according to the utility function

$$
U^{i}(c)=E^{P^{i}}\left(\sum_{t \in N_{+}}\left(\beta^{i}\right)^{t} u^{i}\left(c_{t}\right)\right),
$$

where $\beta^{i} \in(0,1)$ is the intertemporal discount factor, and $u^{i}$ is a strictly increasing, strictly concave, twice-continuously differentiable function. We assume that $u^{i}$ satisfies the Inada condition, namely $\left(u^{i}\right)^{\prime}(c) \mapsto \infty$ as $c \mapsto 0$. Further restrictions on $u^{R}$ are described later.

The budget constraints faced in every history $s_{t}$ by agent $i(i=M, R)$ are

$$
\begin{aligned}
c_{s_{t}}+q_{s_{t}} \theta_{s_{t}} & \leq w_{s_{t}}^{i}+d_{s_{t-1}} \theta_{s_{t-1}} \\
c_{s_{t}} & \geq 0
\end{aligned}
$$

where $s_{t} \hookrightarrow s_{t-1}$ and with the convention that $\theta_{s_{-1}}=d_{s_{-1}}=(0, \ldots, 0)$.

Given the constraints faced by the traders, we also need to rule out the possibility of rolling over any debt through excessive future borrowing, also known as Ponzi's scheme. Consider any vector of prices that is arbitrage-free. Huang and Werner (2004) show that, when a vector of prices $q$ is arbitrage-free, there exists a sequence of positive numbers $\left\{\pi_{s_{t}}\right\}_{s_{t} \in S^{t}, t \in N_{+}}$with $\pi_{s_{0}}=1$ such that

$$
\pi_{s_{t}} q_{s_{t}}^{j}=\pi_{s_{t+1}}
$$


where $s_{t+1}=\left(s_{t}, j\right)$, for every $j(j=1, \ldots, J)$ and $s_{t}\left(s_{t} \in S^{t}\right.$ and $\left.t \in N_{+}\right)$. The sequence of prices $\pi$ is often called the event prices associated with $q$; it obtains independently of the market structure from the no-arbitrage condition. We now assume that every agent cannot borrow more than the present value of her current endowment at such prices. Formally, we assume that for any vector of prices $q$ that is arbitrage-free, every portfolio strategy satisfies the wealth constraints

$$
q_{s_{t}} \theta_{s_{t}} \geq-\frac{1}{\pi_{s_{t}}} \sum_{s_{\tau} \in C\left(s_{t}\right)} \pi_{s_{\tau}} w_{s_{\tau}}^{i} \text { for every } s_{t} .
$$

For every $i(i=M, R)$, we define the budget set $B^{i}(q)$ faced by agent $i$ at prices $q$ as follows. If $q$ is arbitrage-free, the budget set $B^{i}(q)$ is the set of sequences $(c, \theta)$ that satisfy conditions (1)-(4) above. If now the vector of prices has an arbitrage opportunity, define $B^{i}(q)$ as the set of sequences $(c, \theta)$ that satisfy conditions (1)-(2) only. We will see next section that in equilibrium, every vector of prices must be arbitrage-free.

\subsection{Definition of equilibrium}

In this section, the strategies available to the agents and the concept of equilibrium used are defined. A strategy for an agent, in history $s_{t}$, is simply the choice of a sequence of consumptions $c=\left(c_{s_{t}}\right)_{s_{t} \in S^{t}, t \in N_{+}}$and portfolio $\theta=\left(\theta_{s_{t}}\right)_{s_{t} \in S^{t}, t \in N_{+}}$. The market manipulation by agent $M$, captured by the endogeneization of the impact of her trades, is described next in our equilibrium concept.

Before defining it, we first make explicit the demand functions of the market 
for consumption good and assets in every history, as a function of asset prices q. For any vector of assets prices $q$, we denote by $c^{R}(q)=\left[c_{s_{t}}^{R}(q)\right]_{s_{t} \in S^{t}, t \in N_{+}}$ (resp. $\left.\theta^{R}(q)=\left[\theta_{s_{t}}^{R}(q)\right]_{s_{t} \in S^{t}, t \in N_{+}}\right)$the vector of consumption good (resp. assets) demanded by the market, defined as the solutions to the program of maximizing $U^{R}(c)$ subject to $(c, \theta) \in B^{R}(q)$.

We define next a notion of equilibrium for this setting, which we refer to as sequential strategic equilibrium due to the sequential nature of markets. We will show later that it is equivalent to a new concept where all decisions are made in period 0 .

Definition 1 A sequential strategic equilibrium (SSE) is a sequence of prices $\widetilde{q}$ and a sequence of consumptions $\left(\widetilde{c}^{M}, \widetilde{\theta}^{M}\right)$ for the strategic agent such that, taking as given the demand functions $\left(c^{R}, \theta^{R}\right)$, the sequence $\left(\widetilde{c}^{M}, \widetilde{\theta}^{M}, \widetilde{q}\right)$ maximizes the expression $U^{M}(c)$ subject to

$$
\begin{aligned}
& (c, \theta) \in B^{M}(q), \\
& c_{s_{t}}+c_{s_{t}}^{R}(q)=w_{s_{t}} \text { and } \\
& \theta_{s_{t}}+\theta_{s_{t}}^{R}(q)=(0, \ldots, 0) \text { for every } s_{t} .
\end{aligned}
$$

Constraints (6) and (7) above capture the market manipulation. They specify the price formation through market clearing conditions, and are endogenized by the strategic agent. Thus, fully aware of the direct impact of her consumptions and asset holdings on asset prices, the strategic agent directly manipulates the market to maximize her utility function. Moreover, in equilibrium, the 
price-takers behave as usual competitive agents, unaware of the market manipulation. We can also easily check that every equilibrium vector of prices must be arbitrage-free, since otherwise the market would have an infinite demand in at least one history and conditions (6) and (7) would be violated.

The above definition is close to that in Gabszewicz and Vial (1972), where Cournot competition is considered in a general equilibrium framework. In our setting, we consider a monopolist setting quantities while taking the market demands as given, in the spirit of Stackelberg leadership. A different approach can be found in Hens et al. (2004), where price-takers endogenize the market manipulations by large traders. The difference is that, in our approach, the price-takers are unaware of the market manipulation.

\subsection{Survival and accuracy of beliefs}

We now define the concepts of vanishing and surviving along a path for an agent, as well as a measurement of accuracy of belief. Most of these definitions can be found in Sandroni (2000).

Throughout this paper, we focus on the long-run evolution of the consumption of an agent. Sandroni (2000) carries the analysis based on accumulation of wealth in an economy with sequential markets. We prefer to emphasize a study on long-run consumption since it fits best our consumption-based environment. The following definition is central to the paper. 
Definition 2 Agent $i(i=M, R)$ vanishes along a path $s \in S^{\infty}$ if $c_{s_{t}}^{i}$ converges to 0 as $t$ converges to infinity. Agent $i$ survives on a path $s \in S^{\infty}$ if she does not vanish on the path s.

Since the endowment of every agent is uniformly bounded away from 0, an agent can always survive along any path by simply staying in autarchy. It naturally follows that vanishing along a path is a consequence of trading choices.

We next define how "close" an agent's belief is from the true probability measure $P$ of the economy, and therefore how good her predictions are.

Definition 3 Agent $i(i=M, R)$ eventually makes accurate predictions along a path $s \in S^{\infty}\left(s=\left(s_{t}, \ldots\right)\right)$ if $\left\|P_{s_{t}}^{i}-P_{s_{t}}\right\|_{\infty}$ converges to 0 as $t$ converges to infinity, where $\|\cdot\|_{\infty}$ is the standard sup-norm defined on $\Gamma$. Agent $i$ merges with the truth if agent $i$ eventually makes accurate predictions $P-$ a.s.

In words, an agent makes accurate predictions on a path if the conditional probability of her belief, given any finite history along this path, becomes similar in the long-run to the true conditional probability along this path.

\section{Long-run performance of the strategic agent}

In this section, we study the equilibrium long-run behavior of the strategic agent. In particular, we show that depending on her subjective beliefs the strategic agent may well vanish in the long-run. Denote by $\Omega$ the function $\left(u^{R}\right)^{\prime}$, which is invertible since strictly decreasing by assumption. We need further assumptions on this function to carry out our analysis. 
Assumption 1. There exists $X>0$ such that $\Omega(c) \cdot c<X$ for every $c \in[0,2 B]$.

Standard utility functions satisfy Assumption 1, as explained later. This assumption is sufficient to guarantee existence, as shown in Appendix A.

Our second assumption is presented next, its motivation is given in the Introduction.

Assumption 2. There exist $\varepsilon, \gamma>0$ such that for every $x \in[0,2 B+\gamma]$

$$
-\frac{\Omega(x)}{\Omega^{\prime}(x)}>x-B+\varepsilon,
$$

where $B$ is the upper-bound on the endowment process.

The two above assumptions are satisfied by a large class of utility functions. For instance, utility functions of the price-taking agents such as the Napierian logarithm, or $u(x)=x^{\rho}$ for $\rho \in(0,1)$ (only cases to get strict concavity), or also $u(x)=e^{a x}$ for $a<0$, satisfy both Assumptions 1 and 2. From this point on, Assumptions 1-2 are assumed.

We next turn to analyzing the long-run performances of the strategic agent, in particular we are interested in finding cases where she can be outperformed by the representative agent. The next result presents the case where the strategic agent faces a market making accurate predictions and both agents have the same discount factor. This result establishes that the manipulator must also make accurate predictions to avoid vanishing in this case. 
Theorem 1 Assume that $\beta^{R}=\beta^{M}$, and that the representative agent eventually makes accurate predictions. In every equilibrium, and P-a.s.,

a) If the strategic agent does not eventually make accurate predictions on a path $s \in S^{\infty}$ then she will vanish on this path.

b) If the strategic agent eventually makes accurate predictions on a path

$s \in S^{\infty}$ then she will survive on this path.

Proof. See Appendix B.

Theorem 1 is important in order to understand how essential a good knowledge of the uncertainty is. It states that, $P$-a.s, the strategic agent must make accurate predictions in order to survive if the market forecasts accurately and has the same discount factor as the strategic agent. Sandroni gets a similar result for price-taking agents only, so this result seems to be robust to perturbations of a given model. Also, a direct consequence of Theorem 1 is that the strategic agent survives $P^{M}$-a.s; that is, the strategic agent believes that she will survive.

Similar results to Sandroni (2000) linking survival odds and a notion of entropy of beliefs also hold in our setting. With this notion of entropy (capturing the idea that the worse the beliefs the higher the entropy along a path), it can be shown that the manipulator must have a lower entropy of beliefs on a given path than the market to survive on this path. Details are omitted to simplify the exposition. 


\section{Conclusion}

Overall, our work shows that the ability to control all asset prices in a financial market through the impact of one's volume of trade is not a sufficient condition for long-run survival. We show that, provided that some general conditions on market preferences are met, an agent with such market power must make more accurate predictions than the market to survive or else disappears.

At a more abstract level, our work shows that the standard heuristic explanation to the Market Selection Hypothesis, based on the idea that the competitiveness of markets eliminates the less able agents as explained in Alchian (1950), is to be reconsidered. We also show that Pareto-optimality of equilibrium allocations is not a necessary condition for the Market Selection Hypothesis to hold, whereas it is proven to be sufficient in Blume and Easley (1992).

Our result also contrasts the position in Nelson and Winter (1992), where it is argued that market structures may play a critical role in the determinants of long-run survival. Our result shows that behavior consistent with long-run survival are identical in the competitive case and in the non-competitive case. That is, we show that the determinants of long-run survival in both cases must be consistent with the rational and informed maximization of expected returns, as long as market preferences satisfy our assumptions. 


\section{A Existence}

This appendix provides a sufficient condition for existence of a SSE. To derive this result, we first establish some form of equivalence between our sequential framework and an Arrow-Debreu type of framework, and we then establish existence in the later case.

\section{A.1 Equivalent model}

In this section, we show that the above framework is equivalent, in a sense defined later, to a new one where contingent decisions are all made in period 0 in an Arrow-Debreu style. This new framework greatly simplifies the analysis, and we will use it throughout. The proof of equivalence follows closely the proof of the equivalence between Arrow-Debreu equilibria and sequential market equilibria in a competitive setting, as described in Wright (1987).

Denote by $p_{s_{t}}$ the price for delivery of the single consumption good in period $t$ in the finite history $s_{t}\left(s_{t} \in S^{t}\right.$ and $\left.t \in N_{+}\right)$. The new budget set $F^{i}(p)$ of agent $i(i=M, R)$, given a sequence of prices $p=\left(p_{s_{t}}\right)_{s_{t} \in S^{t}, t \in N_{+}}$, is by definition the set of all positive consumption streams $\left(c_{s_{t}}\right)_{s_{t} \in S^{t}, t \in N_{+}}$such that

$$
\sum_{s_{t} \in S^{t}, t \in N_{+}} p_{s_{t}} \cdot c_{s_{t}} \leq \sum_{s_{t} \in S^{t}, t \in N_{+}} p_{s_{t}} \cdot w_{s_{t}}^{i} .
$$

For a sequence of prices $p$, let $\bar{c}^{R}(p)=\left(\bar{c}_{s_{t}}^{R}(p)\right)_{s_{t} \in S^{t}, t \in N_{+}}$be the vector of demand for consumption good for the market; i.e., the vector $\bar{c}^{R}(p)$ maximizes $U^{R}(c)$ subject to $c \in F^{R}(p)$. 
A strategic equilibrium (SE) is then defined to be a sequence of prices $p^{M}$ and a sequence of consumption $c^{M}$ for the strategic agent such that, taking as given the demand functions $\bar{c}^{R}$, the sequence $\left(c^{M}, p^{M}\right)$ maximizes the expression $U^{M}(c)$ subject to

$$
\begin{aligned}
& c \in F^{M}(p), \text { and } \\
& c_{s_{t}}+\bar{c}_{s_{t}}^{R}(p)=w_{s_{t}} \text { for every } s_{t} .
\end{aligned}
$$

A strategic equilibrium is similar to a sequential equilibrium in that the strategic agent endogenizes the effect on prices of her consumption. The difference is that agents now trade in period 0 contingent claims to deliver consumption goods in every history.

The next result shows that the concept of sequential strategic equilibrium under wealth constraint and the concept of strategic equilibrium are equivalent.

Proposition 2 i) Let $\left(c^{M}, p\right)$ be a strategic equilibrium, and define $q_{s_{t}}^{j}=\frac{p_{\left(s_{t}, j\right)}}{p_{s_{t}}}$ for every state $j$ and every $s_{t}$. Then there exits a portfolio strategy $\theta^{M}$ such that $\left(c^{M}, \theta^{M}, q\right)$ is a SSE.

ii) Let $\left(c^{M}, \theta^{M}, q\right)$ be a SSE, and define $p_{s_{t}}=\pi_{s_{t}}$ for every $s_{t}$, where $\pi$ is the system of event prices associated with $q$ as in (3). Then $\left(c^{M}, p\right)$ is a strategic equilibrium.

Proof. The idea of the proof is to show that the agents' budget sets coincide in both concepts, modulo the price transformations specified in the text. We first establish equivalence of budget sets for the representative agent. For every $c$ and 
$p$ such that $c \in F^{R}(p)$, by the main result in Wright (1987), there exists a vector of portfolio $\theta$ such that $(c, \theta) \in B^{R}(q)$ and $(c, \theta)$ satisfies the wealth constraint for the representative agent, where $q_{s_{t}}^{j}=\frac{p_{\left(s_{t}, j\right)}}{p_{s_{t}}}$ for every $s_{t}$ and $j$. Moreover, fix now any asset prices $q$, and any $(c, \theta) \in B^{R}(q)$ satisfying the wealth constraint. Again by the main result in Wright (1987), for the system of prices $p=\pi$, where $\pi$ is associated with $q$ as in Section 2.2, we have that $c \in F^{R}(p)$. This implies that $\bar{c}^{R}(p)=c^{R}(q)$ for every $p$ and corresponding $q$ as defined above. We next turn to the equivalence for the strategic agent. Fix any $c$ and $p$ such that $(c, p)$ satisfies (8) and (9). Define $q$ as above, and also $\theta_{s t}^{M}=-\theta_{s_{t}}^{R}(q)$ for every $s_{t}$. By construction of $c^{R}(q)$ and $\theta^{R}(q)$, and by (9), we have that $\left(c, \theta^{M}\right) \in B^{M}(q)$ and $\left(c, \theta^{M}\right)$ satisfies all the other constraints faced by the strategic agent in a SSE. Fix any $(c, \theta, q)$ satisfying (5)-(7) and the wealth constraint. Conversely, for the system of prices $p=\pi$ and by construction of $\bar{c}^{R}(p)$, it must also be true that $c \in F^{M}(p)$.

Thus we have proved that the budget sets of every agent under the two different settings are identical via the above transformations. It follows that the strategic agent choices in both equilibria are the same, modulo the transformation described in the proposition. The proof is now complete.

From now on, we will carry out the analysis with the concept of strategic equilibrium. The above proposition states that it is always possible to come back to the original framework with sequential markets.

It is important to notice that any equilibrium price in a strategic equilibrium 
must lead to a finite wealth for every agent, since otherwise an agent would have an infinite demand in every history. This issue will play an important role in the proof of existence presented next.

\section{A.2 Existence}

We now prove existence of a SE, with some restrictions on the demand functions of the market as described next.

Theorem 3 Under Assumption 1, there exists a strategic equilibrium.

Before proving this result, we derive an explicit representation of the demand functions from the market. The Inada conditions imply that, for every strictly positive prices set by the strategic agent, the demand of the representative agent in every history is interior. From the problem of the representative agent in a strategic equilibrium, for every sequence of prices $p$ set by the strategic agent there exists a constant $\lambda_{p}$ such that

$$
\left(\beta^{R}\right)^{t} \cdot d P_{s_{t}}^{R} \cdot\left(u^{R}\right)^{\prime}\left(c_{s_{t}}^{R}\right)=\lambda_{p} \cdot p_{s_{t}}
$$

for every $s_{t}$ (the constant $\lambda_{p}$ is the Lagrangian multiplier associated with the program at prices $p)$.

Denote by $\Omega_{s_{t}}$ the function $\left(\beta^{R}\right)^{t} \cdot d P_{s_{t}}^{R} \cdot\left(u^{R}\right)^{\prime}$. It follows that the demand function from the representative agent, in history $s_{t}$, is given by

$$
c_{s_{t}}^{R}\left(p_{s_{t}}\right)=\Omega^{-1}\left(\frac{\lambda_{p} \cdot p_{s_{t}}}{\left(\beta^{R}\right)^{t} \cdot d P_{s_{t}}^{R}}\right) .
$$


We start the proof of Theorem 3 with a technical lemma, giving an upperbound of the wealth the strategic agent can accumulate through market manipulation.

Lemma 4 There exists $\gamma>0$ such that, for every (c, p) satisfying (9), we have that

$$
\sum_{s_{t}} p_{s_{t}}\left(w_{s_{t}}^{M}-c_{s_{t}}\right)<\gamma
$$

Proof. We proceed by way of contradiction. Assume that there exist a sequence of real numbers $\left(\gamma^{n}\right)_{n \geq 0}$ converging to infinity, and a sequence $\left(c^{n}, p^{n}\right)$ satisfying (9) such that

$$
\sum_{s_{t}} p_{s_{t}}^{n}\left(w_{s_{t}}^{M}-c_{s_{t}}^{n}\right)>\gamma^{n}
$$

In particular, relation (11) implies that

$$
\sum_{s_{t}} p_{s_{t}}^{n} w_{s_{t}}^{M}>\gamma^{n}
$$

and since aggregate endowments are bounded away from 0 and infinity, we also have that

$$
\sum_{s_{t}} p_{s_{t}}^{n} w_{s_{t}}^{R}>\gamma^{n}
$$

Moreover, since we have that $c^{n} \in \prod_{s_{t} \in S^{t}, t \in N_{+}}\left[0, w_{s_{t}}\right]$, there exists $\bar{c}$ such that $c^{n}$ converges to $\bar{c}$ modulo the extraction of a subsequence. By (9), the corresponding demands for the representative must satisfy for every $s_{t}$

$$
c_{s t}^{R}\left(p^{n}\right) \rightarrow w_{s_{t}}-\bar{c}_{s_{t}} \leq w_{s_{t}} .
$$


Since, at the limit, the wealth of the representative agent is infinite by (13), this agent must have an infinite demand for some $s_{t}$. This contradicts (14), and the proof is now complete.

With the above lemma, we now prove our theorem. Consider the program which consists of maximizing $U^{M}$ subject to (8) and (9) at period 0 .

Since $U^{M}$ is strictly increasing, it follows that the constraints (8) must hold with equality. By (10), any sequence $c$ satisfying constraints (8) and (9) also satisfies the constraint

$$
\sum_{s_{t} \in S^{t}, t \in N_{+}} \Omega_{s_{t}}\left(w_{s_{t}}-c_{s_{t}}\right) \cdot c_{s_{t}}=\sum_{s_{t} \in S^{t}, t \in N_{+}} \Omega_{s_{t}}\left(w_{s_{t}}-c_{s_{t}}\right) \cdot w_{s_{t}}^{M} .
$$

Clearly, a solution to the program consisting of maximizing $U^{M}$ subject to (15) is a strategic equilibrium. We next show that the set of sequences that satisfy (15) is non-empty and compact for the product topology.

Non-emptiness: the sequence $\left(w_{s_{t}}^{M}\right)_{s_{t} \in S^{t}, t \in N_{+}}$satisfies (15).

Compactness: For every sequence $c \in \prod_{s_{t} \in S^{t}, t \in N_{+}}\left[0, w_{s_{t}}\right]$, consider the functional

$$
\Phi(c) \equiv \sum_{s_{t} \in S^{t}, t \in N_{+}} \Omega_{s_{t}}\left(w_{s_{t}}-c_{s_{t}}\right) \cdot\left(w_{s_{t}}^{M}-c_{s_{t}}\right)
$$

By Lemma 4, we can limit the domain of $\Phi$ to $D \equiv \Phi^{-1}([o, \gamma])$ in our search for equilibrium.

We next show that $\Phi$ restricted on the set $D$ is continuous for the product topology. Fix any arbitrary $\varepsilon>0$, and consider a sequence of consumptions $\left(c^{n}\right)_{n \in N}$ converging to $c$ for the product topology. We next show that there 
exists an integer $n_{0}$ such that, for every $n \geq n_{0}$ we have that $\left|\Phi\left(c^{n}\right)-\Phi(c)\right|<\varepsilon$, proving continuity.

To prove this claim, we break the sum $\left|\Phi\left(c^{n}\right)-\Phi(c)\right|$ in two different parts. In a first step, we show that there exists $t_{0}$ such that

$$
\left|\sum_{s_{t} \in S^{t+t_{0}}, t \in N_{+}}\left[\Omega_{s_{t}}\left(w_{s_{t}}-c_{s_{t}}^{n}\right) \cdot\left(w_{s t}^{M}-c_{s_{t}}^{n}\right)-\Omega_{s_{t}}\left(w_{s_{t}}-c_{s_{t}}\right) \cdot\left(w_{s_{t}}^{M}-c_{s_{t}}\right)\right]\right|<\frac{\varepsilon}{2}
$$

for every $n$. Then, we use the continuity of $\Omega_{s_{t}}$, for the first $\sum_{t=0}^{t_{0}} L^{t}$ events $s_{t},{ }^{6}$ to show that the difference $\left|\Phi\left(c^{n}\right)-\Phi(c)\right|$ restricted to the first $\sum_{t=0}^{t_{0}} L^{t}$ events can be made arbitrarily small for $n$ large enough.

Fix first any arbitrary $x, y \in \prod_{s_{t} \in S^{t}, t \in N_{+}}\left[0, w_{s_{t}}\right]$, and any period $p$. We have that

$$
\begin{aligned}
&\left|\sum_{s_{t} \in S^{t+p}, t \in N_{+}}\left[\begin{array}{c}
\Omega_{s_{t}}\left(w_{s_{t}}-x_{s_{t}}\right) \cdot\left(w_{s_{t}}^{M}-x_{s_{t}}\right) \\
-\Omega_{s_{t}}\left(w_{s_{t}}-y_{s_{t}}\right) \cdot\left(w_{s_{t}}^{M}-y_{s_{t}}\right)
\end{array}\right]\right| \\
&<\left|\sum_{s_{t} \in S^{t+p}, t \in N_{+}}\left[\begin{array}{c}
\Omega_{s_{t}}\left(w_{s_{t}}-x_{s_{t}}\right) \cdot\left(w_{s_{t}}^{M}-x_{s_{t}}\right) \\
+\Omega_{s_{t}}\left(w_{s_{t}}-y_{s_{t}}\right) \cdot\left(w_{s_{t}}^{M}-y_{s_{t}}\right)
\end{array}\right]\right| \\
&<\left|\sum_{s_{t} \in S^{t+p}, t \in N_{+}}\left[\begin{array}{c}
\Omega_{s_{t}}\left(w_{s_{t}}-x_{s_{t}}\right) \cdot\left(w_{s_{t}}-x_{s_{t}}\right) \\
+\Omega_{s_{t}}\left(w_{s_{t}}-y_{s_{t}}\right) \cdot\left(w_{s_{t}}-y_{s_{t}}\right)
\end{array}\right]\right| \\
& \sum_{2 X}\left(\beta_{s_{t} \in S^{t+p}, t \in N_{+}} \sum_{s_{s_{t}} .}\right.
\end{aligned}
$$

\footnotetext{
${ }^{6}$ Recall that in any period $t$, there are $L^{t}$ possible events, and thus between period 0 and $t_{0}$ there are $\sum_{t=0}^{t_{0}} L^{t}$ events.
} 
Since $\lim \sum_{s_{t} \in S^{t+p}, t \in N_{+}}\left(\beta^{R}\right)^{t} \cdot d P_{s_{t}}=0$, it follows that there exists a time $t_{0}$ such that, for every $x$ and $y$,

$\left|\sum_{s_{t} \in S^{t+t_{0}}, t \in N_{+}}\left[\Omega_{s_{t}}\left(w_{s_{t}}-x_{s_{t}}\right) \cdot\left(w_{s_{t}}^{M}-x_{s_{t}}\right)-\Omega_{s_{t}}\left(w_{s_{t}}-y_{s_{t}}\right) \cdot\left(w_{s_{t}}^{M}-y_{s_{t}}\right)\right]\right|<\frac{\varepsilon}{2}$.

We can apply this result to the sequences $\left(c^{n}\right)_{n \in N}$ and $c$ to get for every $n$ that

$\left|\sum_{s_{t} \in S^{t+t_{0}, t \in N_{+}}}\left[\Omega_{s_{t}}\left(w_{s_{t}}-c_{s_{t}}^{n}\right) \cdot\left(w_{s_{t}}^{M}-c_{s_{t}}^{n}\right)-\Omega_{s_{t}}\left(w_{s_{t}}-c_{s_{t}}\right) \cdot\left(w_{s_{t}}^{M}-c_{s_{t}}\right)\right]\right|<\frac{\varepsilon}{2}$.

Moreover, by continuity of the function $\Omega_{s_{t}}$, we have for every history $s_{t}$ that

$$
\Omega_{s_{t}}\left(w_{s_{t}}-c_{s_{t}}^{n}\right) \cdot\left(w_{s_{t}}^{M}-c_{s_{t}}^{n}\right) \rightarrow \Omega_{s_{t}}\left(w_{s_{t}}-c_{s_{t}}\right) \cdot\left(w_{s_{t}}^{M}-c_{s_{t}}\right) .
$$

Therefore, for every $s_{t}$ such that $t \leq t_{0}$, there exists $n_{s_{t}} \in \mathbb{N}$ such that for every $n \geq n_{s_{t}}$, the following holds:

$$
\left|\Omega_{s_{t}}\left(w_{s_{t}}-c_{s_{t}}^{n}\right) \cdot\left(w_{s_{t}}^{M}-c_{s_{t}}^{n}\right)-\Omega_{s_{t}}\left(w_{s_{t}}-c_{s_{t}}\right) \cdot\left(w_{s_{t}}^{M}-c_{s_{t}}\right)\right|<\frac{\varepsilon}{2 \cdot \sum_{t=0}^{t_{0}} L^{t}} .
$$

Define now $n_{0}=\max _{s_{t} \in S^{t}, 0 \leq t \leq t_{0}} n_{s_{t}}$. For every $n \geq n_{0}$, we get that:

$$
\begin{aligned}
& \left|\Phi\left(c^{n}\right)-\Phi(c)\right| \\
& =\left|\begin{array}{c}
\sum_{s_{t} \in S^{t}, 0 \leq t \leq t_{0}}\left[\Omega_{s_{t}}\left(w_{s_{t}}-c_{s_{t}}^{n}\right) \cdot\left(w_{s_{t}}^{M}-c_{s_{t}}^{n}\right)-\Omega_{s_{t}}\left(w_{s_{t}}-c_{s_{t}}\right) \cdot\left(w_{s_{t}}^{M}-c_{s_{t}}\right)\right] \\
+\sum_{s_{t} \in S^{t+t_{0}, t \in N_{+}}}\left[\Omega_{s_{t}}\left(w_{s_{t}}-c_{s_{t}}^{n}\right) \cdot\left(w_{s_{t}}^{M}-c_{s_{t}}^{n}\right)-\Omega_{s_{t}}\left(w_{s_{t}}-c_{s_{t}}\right) \cdot\left(w_{s_{t}}^{M}-c_{s_{t}}\right)\right]
\end{array}\right| \\
& <\left|\sum_{s_{t} \in S^{t}, 0 \leq t \leq t_{0}}\left[\Omega_{s_{t}}\left(w_{s_{t}}-c_{s_{t}}^{n}\right) \cdot\left(w_{s_{t}}^{M}-c_{s_{t}}^{n}\right)-\Omega_{s_{t}}\left(w_{s_{t}}-c_{s_{t}}\right) \cdot\left(w_{s_{t}}^{M}-c_{s_{t}}\right)\right]\right| \\
& +\left|\sum_{s_{t} \in S^{t+t_{0}, t \in N_{+}}}\left[\Omega_{s_{t}}\left(w_{s_{t}}-c_{s_{t}}^{n}\right) \cdot\left(w_{s_{t}}^{M}-c_{s_{t}}^{n}\right)-\Omega_{s_{t}}\left(w_{s_{t}}-c_{s_{t}}\right) \cdot\left(w_{s_{t}}^{M}-c_{s_{t}}\right)\right]\right|
\end{aligned}
$$




$$
\begin{aligned}
& <\sum_{s_{t} \in S^{t}, 0 \leq t \leq t_{0}}\left(\frac{\varepsilon}{2 \cdot \sum_{t=0}^{t_{0}} L^{t}}\right)+\frac{\varepsilon}{2} \\
& =\frac{\varepsilon}{2}+\frac{\varepsilon}{2}=\varepsilon .
\end{aligned}
$$

This proves continuity of the function $\Phi$ restricted on $D$ for the product topology.

The set of sequences $c$ that satisfy (15) is also $\Phi^{-1}(\{0\})$ by construction. Since the functional $\Phi$ is continuous on the set $D$ for the product topology, the set $\Phi^{-1}(\{0\})$ is closed in the compact set $\prod_{s_{t} \in S^{t}, t \in N_{+}}\left[0, w_{s_{t}}\right]$, and compactness follows.

Following standard arguments, we have that $U^{M}$ is also continuous for the product topology on the set $\prod_{s_{t} \in S^{t}, t \in N_{+}}\left[0, w_{s_{t}}\right]$.

Therefore, the program consisting of maximizing $U^{M}$ subject to (15) has a solution, and a strategic equilibrium exists.

Existence of a SSE follows from the above result and Proposition 2. One can notice that a direct use of dynamic programming tools would not be of any particular help when proving existence of a SSE. The reason is that the statedependent realization of endowment, and the state-dependent demand functions from the market, change the value function of the strategic agent in every finite history. Our proof of existence, which uses the equivalence between SSE and SE, allows to avoid this issue. 


\section{B Proof of Theorem 1}

The following three lemmas are central to proving Theorem 1, and they are the keys to understanding the whole analysis. The first lemma is Lemma 3 in Sandroni (2000), which is independent of the market structure.

Lemma 5 For every agent $i(i=M, R)$ and $P$-a.s, it is true that

$$
\infty>\lim _{t \longrightarrow \infty} \frac{d P_{t}^{i}}{d P_{t}} \geq 0
$$

Moreover, agent $i(i=M, R)$ eventually makes accurate predictions on a path $s \in S^{\infty}(P-a . s)$ if and only if

$$
\infty>\lim _{t \rightarrow \infty} \frac{d P_{t}^{i}}{d P_{t}}>0
$$

The above lemma, as Sandroni points out, states that almost surely an agent $i$ eventually makes accurate predictions on a path $s$ if and only the probability that agent $i$ assigns to $s_{t}$ does not become arbitrary smaller than the true probability of $s_{t}$ as $t$ goes to infinity. The next lemma gives a necessary condition that the equilibrium values must satisfy.

Lemma 6 In every equilibrium, there exists a strictly positive constant $\Theta>0$ such that, for every $s \in S^{\infty}$, the equilibrium consumption variables satisfy

$$
\Theta\left(\frac{\beta^{M}}{\beta^{R}}\right)^{t} \frac{d P_{t}^{M}}{d P_{t}^{R}} u^{\prime}\left(c_{s_{t}}^{M}\right)=\left(w_{s_{t}}^{M}-c_{s_{t}}^{M}\right) \Omega^{\prime}\left(c_{s_{t}}^{M}\right)+\Omega\left(c_{s_{t}}^{M}\right) .
$$

By Assumption 2, the right-hand side of (16) is necessarily positive and bounded away from 0 . The above lemma shows that some restrictions on the utility functions are needed to get interior solutions to the program faced by the strategic 
agent. However, usual utility functions in economic theory, such as the Napierian logarithm or any function $u(x)=x^{\rho}$ for any $\rho<1$, can generate an interior solution satisfying (16).

Proof. Consider the problem of maximizing $U^{M}$ subject to (15). The firstorder conditions to this program directly gives (16), where the constant $\Theta$ is the Lagrange multiplier associated with constraint (15). This multiplier must also be strictly positive since (15) holds with equality. This finishes the proof.

The next lemma is a direct consequence of Lemma 5 and Lemma 6 .

Lemma 7 In every equilibrium and for every path $s \in S^{\infty}$,

i) if beliefs are such that

$$
\lim _{t \rightarrow \infty}\left(\frac{\beta^{M}}{\beta^{R}}\right)^{t} \frac{d P_{t}^{M}}{d P_{t}^{R}}=0
$$

then the strategic agent will vanish on the path s.

ii) If there exists $\varepsilon>0$ such that

$$
\left(\frac{\beta^{M}}{\beta^{R}}\right)^{t} \frac{d P_{t}^{M}}{d P_{t}^{R}}>\varepsilon \text { for every } t \in N_{+},
$$

then the strategic agent will survive on the path s.

Proof. We first prove i) above. Pick any equilibrium and any path $s \in S^{\infty}$, and assume that $\left(\frac{\beta^{M}}{\beta^{R}}\right)^{t} \frac{d P_{t}^{M}}{d P_{t}^{R}}$ is 0. Consider now Eq. (16), which rewrites as

$$
\Theta\left(\frac{\beta^{M}}{\beta^{R}}\right)^{t} \frac{d P_{t}^{M}}{d P_{t}^{R}}=\frac{\left(w_{s_{t}}^{M}-c_{s_{t}}^{M}\right) \Omega^{\prime}\left(c_{s_{t}}^{R}\right)+\Omega\left(c_{s_{t}}^{R}\right)}{\left(u^{M}\right)^{\prime}\left(c_{s_{t}}^{M}\right)} .
$$

By Assumption 2, the numerator in the above expression is bounded away from 0 . Therefore it must be true that $u^{\prime}\left(c_{s_{t}}^{M}\right)$ converges to infinity, and by 
assumptions on $u^{M}$ the sequence $\left(c_{s_{t}}^{M}\right)_{s_{t} \in S^{t}, t \in N_{+}}$must converge to 0 as $t$ converges to infinity. This proves that the strategic agent vanishes along this path $s$.

To prove ii), we proceed by way of contradiction. Assume that the strategic agent vanishes along the path $s$. Then from (16) it must true that

$$
\Theta \varepsilon<\frac{\left(w_{s_{t}}^{M}-c_{s_{t}}^{M}\right) \Omega^{\prime}\left(c_{s_{t}}^{R}\right)+\Omega\left(c_{s_{t}}^{R}\right)}{\left(u^{M}\right)^{\prime}\left(c_{s_{t}}^{M}\right)} .
$$

Since the strategic agent vanishes along the path $s$, it follows that $\left(u^{M}\right)^{\prime}\left(c_{s_{t}}^{M}\right)$ converges to infinity since $c_{s_{t}}^{M}$ converges to 0 . Therefore, for (17) to hold, it must be true that the numerator in its right-hand side converges to infinity.

Also for $t$ large enough it is true that $w_{s_{t}}^{M}-c_{s_{t}}^{M}>0$, thus $\left(w_{s_{t}}^{M}-c_{s_{t}}^{M}\right) \Omega^{\prime}\left(c_{s_{t}}^{R}\right)<0$ by strict concavity of $u^{R}$. Thus, it must be true that $\Omega\left(c_{s_{t}}^{R}\right)$ converges to infinity for (17) to hold. It directly follows, by the Inada conditions, that $c_{s_{t}}^{R}$ converges to 0 , violating the fact that aggregate endowments are bounded away from 0 along the path $s$. This is a contradiction, and the proof is now complete.

Intuitively, the above lemma says that, when all the agents have the same intertemporal discount factor, if the strategic agent believes that a path is less likely to occur than the market, then she will set the manipulation so as to consume less and less on this path. Eventually, her optimal hedging plans will make her consume nothing asymptotically along this path. However, if now the strategic agent does not believe that a path is less likely to occur than the market, then she will choose to consume along this path a quantity of good that is uniformly bounded away from 0 . 
With the three above lemmas, we now prove Theorem 1 as follows. Consider any set $A \in \Gamma$ as described in Lemma 5. This set is such that $P(A)=1$ and the properties in Lemma 5 hold for any path in $A$. Pick any $s \in A$ such that the representative agent eventually makes accurate predictions on $s$.

We now prove a) for any $s \in A$. In this case, from Lemma 5 we have that $\lim \frac{d P_{t}}{d P_{t}^{R}}<\infty$. Since the strategic agent does not make accurate predictions on $s$, and again from Lemma 5, it follows that $\lim \frac{d P_{t}^{M}}{d P_{t}}=0$. Therefore $\lim \frac{d P_{t}^{M}}{d P_{t}^{R}}=0$, and by Lemma 7 the strategic agent vanishes along the path $s$.

We next prove b) for any path $s \in A$. In this case, we have from Lemma 5 that $\lim \frac{d P_{t}}{d P_{t}^{i}}>0$ for every agent $i(i=M, R)$. It follows that $\lim \frac{d P_{t}^{M}}{d P_{t}^{R}}>0$, and by Lemma 7 the strategic agent survives on the path $s$. The proof is now complete.

\section{References}

[1] Alchian, A.: Uncertainty, evolution and economic theory. Journal of Political Economy 58, 211-221 (1950)

[2] Blume, L., Lawrence, Easley, D.: Evolution and market behavior. Journal of Economic Theory 58, 9-40 (1992)

[3] Blume, L., Easley, D.: If you're so smart, why aren't you rich? Belief selection in complete and incomplete markets. Cowles Foundation No 1319 (2004)

[4] Debreu, G.: Theory of Value. Willey, New-York 1959 
[5] Friedman, M.: Essays in Positive Economics. University of Chicago Press, Chicago 1953

[6] Gabszewicz, J., Vial, J.-P.: Oligopoly 'à la Cournot' in general equilibrium analysis. Journal of Economic Theory 4, 381-400 (1972)

[7] Hens, T., Reimann, S., Vogt, B.: Nash competitive equilibria and the twoperiod fund separation. Journal of Mathematical Economics 40, 321-346 (2004)

[8] Huang, K., Werner, J.: Implementing Arrow-Debreu equilibria by trading infinitely-lived securities. Economic Theory 24, 603-623 (2004)

[9] Nelson, R., Winter, S.: An Evolutionary Theory of Economic Changes. The Belknap Press of Harvard University Press, Cambridge 1982

[10] Sandroni, A.: Do markets favor agents able to make accurate predictions? Econometrica 68, 1303-1341 (2000)

[11] Stockey, N. Lucas, R.: Recursive Methods in Economic Dynamics. Harvard University Press, Cambridge 1996

[12] Wright, R.: Market structure and competitive equilibrium in dynamic economic models. Journal of Economic Theory 41, 189-201 (1987) 